$90.3 \%(140 / 155)$ in pSS, 92.3\% (36/39) in MCTD, 93.4\% (128/137) in PBC and $49.1 \%(28 / 57)$ in the AlH group. The positive rate of ANA in the health subjects was $11.5 \%(123 / 1073)$, see Figure 1.

Totally, the positive rate of ANA in the AID group was $80.1 \%$ (2967/ 3704). The karyotypes were detected as homogeneous type (15.8\% (586/ $3704))$, nuclear particle type $(49.7 \%$ (1840/3704)), cytoplasmic particle type $(5.3 \%(196 / 3704))$, mixed type $(2.8 \%$ (91/3704)) which was mainly composed of nuclear membrane/cytoplasmic granules, more common in PBC. See Table 1. The karyotype of ANA in the health group was mainly homozygous $(7.0 \%(75 / 1073))$.

\begin{tabular}{|c|c|c|c|c|c|c|c|c|c|c|}
\hline \multirow[b]{2}{*}{ Group } & \multirow[b]{2}{*}{$\mathrm{n}$} & \multicolumn{8}{|c|}{ ANA karyotypes } & \multirow{2}{*}{$\underset{\text { negat ivity }}{\stackrel{A N A}{1}}$} \\
\hline & & Homogeneous & particle & centromere & nucleolar & $\begin{array}{c}\text { cytoplasmic } \\
\text { granularar }\end{array}$ & $\begin{array}{l}\text { nuclear } \\
\text { nembrane }\end{array}$ & nixed & other & \\
\hline SLE & 2034 & $337(16.6)^{\circ}$ & $1328(65.3)$ & $23(1.1)$ & $28(1.4)$ & $86(4,2)$ & $9(0.4)$ & $23(1.1)$ & $10(0.5)$ & $190(9.3)$ \\
\hline RA & 973 & $186(19,1)$ & $255(26.2)$ & $16(1.6)$ & $10(1.0)$ & $49(5,0)$ & $1(0.1)$ & $0(0.0)$ & $8(0.8)$ & $448(46.0)$ \\
\hline ss & 309 & $34(11.0)$ & $181(58.6)$ & $13(4.2)$ & $8(2.6)$ & $15(4.9)$ & $3(1.0)$ & $10(3.2)$ & $3(1.0)$ & $42(13.6)$ \\
\hline PSS & 155 & $22(14.2)$ & $27(17.4)$ & $28(18.1)$ & $42(27.1)$ & $8(5.2)$ & $2(1.3)$ & $9(5.8)$ & $2(1.3)$ & $15(9.7)$ \\
\hline MCTD & 39 & $3(7.7)$ & $30(76.9)$ & $1(2.6)$ & $0(0.0)$ & $2(5.1)$ & $0(0.0)$ & $0(0.0)$ & $0(0.0)$ & $3(7.7)$ \\
\hline PBC & 137 & $1(0.7)$ & $6(4,4)$ & $17(12.4)$ & $2(1.5)$ & $36(26.3)$ & $15(10.9)$ & $16(33.6)$ & $5(3.6)$ & $9(6,6)$ \\
\hline AIH & 57 & $3(5.3)$ & $13(22.8)$ & $7(12.3)$ & $0(0.0)$ & $0(0,0)$ & $0(0.0)$ & $3(5.3)$ & $2(3.5)$ & $29(50.9)$ \\
\hline AID & 3704 & $586(15.8)$ & $1840(49.7)$ & $105(2.8)$ & $90(2.4)$ & $196(5.3)$ & $30(0.8)$ & $91(2.5)$ & $30(0.8)$ & $736(19.9)$ \\
\hline $\begin{array}{l}\text { Heal thy } \\
\text { subjects }\end{array}$ & 1073 & $14(1.3)$ & $75(7.0)$ & $3(0.3)$ & $10(0.9)$ & $23(2.2)$ & $1(0.1)$ & $2(0.2)$ & $3(0.3)$ & $942(88.5)$ \\
\hline
\end{tabular}

Figure 1 ANA positive rate in difference AID patients and healthy subjects

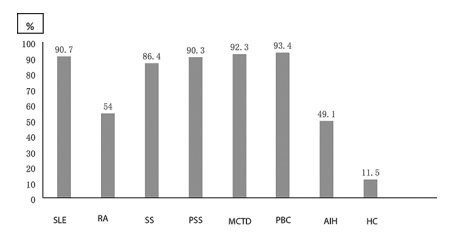

Conclusion: There are differences in ANA positive rates among patients with different AIDs. Some mixed karyotype analyses may provide important evidence for the diagnosis of PBC and AlH. Healthy subjects can have partial ANA positivity which is predominantly homogenous.

Disclosure of Interests: None declared

DOI: 10.1136/annrheumdis-2019-eular.8381

\section{THU0611 SERUM HIGHLY-SENSITIVE CARDIAC TROPONIN-I AND ANTI-BETA2-GLYCOPROTEIN-1 IGA ANTIBODIES AT BASELINE PREDICT CORONARY PLAQUE PROGRESSION IN RHEUMATOID ARTHRITIS}

George Karpouzas $^{1}$, Sarah Ormseth ${ }^{1}$, Elizabeth Hernandez ${ }^{1}$, Joel Estis ${ }^{2}$, John Todd ${ }^{2}$, Matthew Budoff 3 . ${ }^{1}$ Harbor UCLA Medical Center, Rheumatology, West Carson, United States of America; ${ }^{2}$ Singulex, Inc., Alameda, United States of America; ${ }^{3}$ Harbor UCLA Medical Center, Cardiology, West Carson, United States of America

Background: We recently reported that serum levels of highly-sensitive cardiac troponin-I (hs-cTnl)- a specific structural myocardial biomarkerassociated with occult coronary plaque presence, burden and long-term cardiovascular events in patients with rheumatoid arthritis (RA). We further demonstrated that presence of $\lg \mathrm{A}$ antibodies against beta2-glycoprotein-1 (a-b2GPI-IgA, an apolipoprotein readily expressed in human atherosclerotic plaque) associated with baseline coronary artery calcium (CAC) scores and independently predicted CAC progression in patients with RA.

Objectives: We here explored whether baseline evaluation of both hscTnl and a-b2GPI-IgA better predicts CAC progression than either of them in isolation.

Methods: Ninety five participants with a baseline plaque evaluation by coronary computed tomography angiography (CCTA) underwent follow-up assessment within $6.9 \pm 0.3$ years. Coronary artery calcium (CAC) was quantified by the Agatston method. Hs-cTnl and a-b2GPI IgA Ab were assessed on the day of baseline CCTA; the latter were reconfirmed 12 weeks later, if positive. CAC change was evaluated with the MESA method as the natural logarithm plus 25 difference $\left[\left(\ln \mathrm{CAC}_{(\text {follow-up })}+25\right)\right.$ - (In $\left.\left.\mathrm{CAC}_{\text {(baseline) }}+25\right)\right]$. Generalized linear models evaluated the effect of hs-cTnl, a-b2GPI-IgA and their interaction on CAC change. Models were adjusted for age, hypertension, waist-to height ratio (obesity indicator), cumulative inflammatory burden (time-averaged C-Reactive protein), total prednisone dose and duration of statin exposure from baseline to follow-up scan

Results: Baseline hs-cTnl was higher in a-b2GPI-lgA positive compared to negative patients [median (IQR) of 1.9 (1.6-2.6) vs. $1.4 \quad(1.3-1.7)$ respectively, $p=0.043$ ]. Hs-cTnl alone did not independently predict CAC change $(\beta=0.214, p=0.132)$ in the multivariable model whereas a-b2GPI$\lg A$ presence did $(\beta=0.454, p=0.003)$. There was a significant interaction between hs-cTnl and a-b2GPI-IgA on CAC progression (Wald Chisquare=4.19, $\mathrm{p}=0.041)$; high hs-cTnl $(>1.5 \mathrm{pg} / \mathrm{ml})$ predicted significantly greater CAC change in a-b2GPI-IgA positive patients but not in negative ones [estimated marginal mean difference $(I Q R)=0.36 \quad(0.12-0.59)$, $p=0.003$ ) and figure 1].

Conclusion: Baseline hs-cTnl in isolation is not predictive of CAC progression in patients with RA; however, in the context of a-b2GPI-IgA presence, high hs-cTnl independently predicts significant increase in coronary atherosclerosis burden.

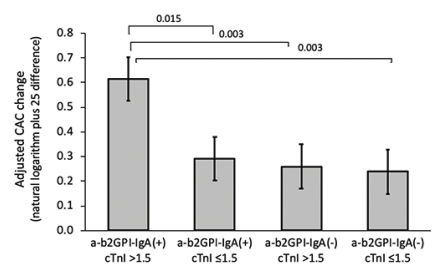

Disclosure of Interests: George Karpouzas Grant/research support from Pfizer, Consultant for: Sanofi-Genzyme-Regeneron, Janssen, Roche-Genentech, Pfizer, Speakers bureau: BMS, Sanofi-Genzyme-Regeneron, Janssen, Roche-Genentech, Sarah Ormseth: None declared, Elizabeth Hernandez: None declared, Joel Estis Employee of: Singulex, John Todd Employee of: Singulex, Matthew Budoff: None declared DOI: 10.1136/annrheumdis-2019-eular.6767

\section{THU0612 KNEE JOINT PAIN IN AN ELDERLY, HEALTHY POPULATION IS ASSOCIATED WITH INFLAMMATORY ARTICULAR AND ENTHESEAL CHANGES DETECTED BY ULTRASOUND}

David Simon ${ }^{1}$, Arnd Kleyer ${ }^{1}$, Antonella Adinolfi ${ }^{2}$, Johann Willeit ${ }^{3}$, Stefan Kiechl ${ }^{3}$, Nina Gasperi ${ }^{4}$, Georg Schett ${ }^{1}$, Christian Dejaco ${ }^{4}$, Annamaria lagnocco ${ }^{5}$, MariaAntonietta D'agostino ${ }^{6} .{ }^{1}$ Friedrich-Alexander University Erlangen-Nuremberg (FAU) and Universitätsklinikum Erlangen, Erlangen, Germany; ${ }^{2}$ University of Siena, Department of Rheumatology, Policlinico Le Scotte, Siena, Italy; ${ }^{3}$ Medical University of Innsbruck, Department of Neurology, Innsbruck, Austria; ${ }^{4}$ Department of Rheumatology, Hospital of Bruneck, Bruneck, Italy, Department of Rheumatology and Immunology, Medical University Graz, Graz, Austria, Graz, Austria; ${ }^{5}$ Università degli Studi di Torino, Dipartimento Scienze Cliniche e Biologiche, Torino, Italy; ${ }^{6}$ Hôpital Ambroise Paré, Boulogne-Billancourt, Department of Rheumatology, APHP, Paris, France

Background: Knee pain is highly prevalent in elderly and influences quality of life. Pathogenesis of knee pain in such population is commonly related to osteoarthritis $(\mathrm{OA})$. It is yet unclear however, which pathological lesions in the knee contribute most to symptomatic disease.

Objectives: To define the articular and enthesal inflammatory and struc tural lesions that contribute to knee pain in an elderly healthy population using Power Doppler ultrasound (PDUS)

Methods: All subjects ( $\geq 65$ years) were part of the prospective long-term population-based Bruneck Study (1) and received a clinical and ultrasound investigation of both knees. Ultrasound was performed by an independent investigator unaware of clinical symptoms. Knee entheses (quadriceps insertion, proximal and distal patella insertion) and joint cavity were assessed. Demographic variables were recorded in all individuals Pain sensation during knee palpation was collected and participants were asked to complete a standardized pain questionnaire for knee joints (Knee injury and osteoarthritis outcome score [KOOS], question P1-P9) (2). Joint changes (synovial hypertrophy, power doppler (PD) signal, joint effusion, baker cysts, osteophytes) were assessed using a GE Logic E 